\title{
El patrón de movimiento en el swing de golf en jóvenes promesas
}

\author{
Cristina lópez de Subijana, Raquel de Antonio, Daniel Juarez y Enrique Navarro \\ Universidad de Alcalá. Área de Educación Física, CAFAD. Departamento de Psicopedagogía y Educación Física. Alcalá. España. \\ Universidad Politécnica de Madrid. Instituto Nacional de Educación Física. Madrid. España.
}

\section{RESUMEN}

Introducción: El swing de golf es una destreza que podríamos catalogar de golpeo de velocidad donde el objetivo es que la cara del palo alcance la máxima velocidad en el momento del impacto.

Objetivos: Analizar la secuencia temporal del swing de golf y determinar su patrón de movimiento.

Métodos: Se han analizado biomecánicamente 10 golpeos de 4 jugadores promesas, mediante el sistema de fotogrametría 3D Vicon Oxford Metrics ${ }^{\circledR}$ de captura automática. Resultados: En primer lugar se ha podido comprobar que los valores absolutos de velocidades lineales eran mayores en los chicos que en las chicas. El análisis de las 2 fases del swing indica que la fase de aceleración downswing presenta una variabilidad menor que el backswing. El análisis de la secuencia temporal de máximos alcanzados en las velocidades lineales de los segmentos muestra que sus acciones siguen un orden diferente en función del sexo. La secuencia en los chicos empieza por el movimiento de caderas y sigue con el codo izquierdo, el hombro izquierdo y el palo; en las chicas, las caderas y el codo izquierdo actúan simultáneamente seguidos del hombro izquierdo y del palo. En cambio, las secuencias de velocidades angulares encontradas seguían en ambos sexos el mismo orden: primero el giro de caderas, luego el giro de hombros y por último la aceleración del palo. Esta secuencia de acciones se encontraba más separada en el tiempo en los hombres que en las mujeres.

Conclusiones: Se hallaron los patrones del movimiento mediante fórmulas discriminantes en ambos sexos, capaces de predecir si el golpeo sería "bueno" o "malo" siguiendo el criterio de rendimiento marcado en la velocidad de la cabeza del palo.

PALABRAS CLAVE: Biomecánica. Golpeo. Golf. Rendimiento. Patrón de movimiento.

\begin{abstract}
Introduction: The golf swing is a skill that could be classified as high velocity hitting in which the main goal is that the head of the golf club reaches its maximum velocity at the moment of impact.

Aims: To analyze the timing sequence of the golf swing and to identify the movement pattern of this skill.

Methods: Ten golf swings executed by four young players were biomechanically analyzed. Automatic capture with $\mathrm{Vi-}$ con Oxford Metrics ${ }^{\circledR}$ was used.

Results: The absolute values of the linear velocities were higher in men than in women. In the interval analysis, the downswing showed less variation than the backswing. Analysis of the maximum speed timing sequence revealed a different order depending on gender. In men, the sequence started with the hip movement, followed by the left elbow, left shoulder and the club head. In women, the hips and left elbow moved simultaneously, followed by the left shoulder and the club head. In contrast, the angular velocity sequences followed the same order in both genders: first the hip turn, then the shoulder turn, and finally the golf club head acceleration. These key events were more separated in the time line in men than in women.

Conclusions: Movement patterns were found through discriminatory formulae in both genders, which were able to predict whether the golf swing was "good" or "bad", taking the club head speed as the performance criterion.
\end{abstract}

KEY WORDS: Biomechanics. Hitting. Golf. Athletic performance. Movement pattern. 


\section{INTRODUCCIÓN}

El swing en golf es una destreza que podríamos catalogar de golpeo de velocidad, donde el objetivo es que la cara del palo alcance la máxima velocidad en el momento del impacto ${ }^{1}$. La eficacia de cada golpeo se basa en la precisión y en la distancia alcanzada $^{2}$. El control de la técnica es un requisito fundamental para realizar una óptima ejecución. En función de la distancia deseada, el golfista elegirá un tipo de palo u otro, hierro o madera.

Los instantes más importantes en el swing de golf son el stance o posición inicial, el top of backswing, la pausa al final de la preparación y el impacto ${ }^{3}$. Las fases del golpeo son el backswing, que es la fase desde el stance hasta el top of backswing; el downswing, que va desde el top of backswing hasta el impacto y es, por tanto, la fase de aceleración, y el followthrough, que va desde el impacto hasta que cesa el movimiento. El middownsuing es el instante en el que el eje longitudinal del palo se sitúa paralelo a la superficie (fig. 1).

La fase analizada en este estudio es la fase de aceleración, el downswing. Cochran y Stobbs ${ }^{4}$ indicaron que el backswing tenía una duración en golfistas profesionales de 0,82 y $0,23 \mathrm{~s}$ en el downswing. Burden et $\mathrm{al}^{5}$ registraron 0,95 y $0,26 \mathrm{~s}$, respectivamente; resultados similares de downswing $(0,23$ y $0,21 \mathrm{~s})$ fueron obtenidos por Milburn ${ }^{6}$ y Neal y Wilson?

Las muestras de los estudios previos son generalmente de sexo masculino y con edades superiores a los 30 años. Únicamente Coleman y Anderson ${ }^{8}$ y Egret et al ${ }^{9}$ se basaron en jugadores jóvenes (alrededor de 20 años). Egret et $\mathrm{a}^{9}$ realizaron un análisis de los ángulos alcanzados en las fases del swing y registraron las velocidades máximas en la punta del palo, que fueron de $40,1 \mathrm{~m} / \mathrm{s}$ en los hombres y de $37,7 \mathrm{~m} / \mathrm{s}$ en las mujeres. Ambos sexos utilizaron un driver. Coleman y Anderson ${ }^{8}$ analizaron el plano de golpeo con diferentes tipos de palos: un driver, un hierro $5 \mathrm{y}$ un wedge.

El principio de "cadena cinética" es un concepto que se basa en que "la producción de una gran velocidad en un extremo distal (en este caso la cara del palo de golf) implica el uso de las aceleraciones y deceleraciones de los segmentos adyacentes, aplicados de una forma secuencial, de más masivo a más concreto y del segmento más fijo al más libre"1. En este caso, la secuencia de acciones debería producirse mostrando primero aceleraciones de los miembros inferiores, luego del tronco, luego de los miembros superiores y por último de la cara de la cabeza del palo.

La secuencia de velocidades analizada por Milburn ${ }^{6}$ estableció un orden de acciones en la fase de aceleración que indi- caba cómo primero actuaba el brazo izquierdo, a continuación la muñeca izquierda y por último el palo. Nesbit y Serrano ${ }^{10}$ expresaron el timing o secuencia de máximos con una función matemática. La muestra utilizada estaba formada por 4 sujetos de diferentes niveles, y cada uno de ellos mostró una función diferente. Sólo un sujeto era de alto nivel. La secuencia de máximos de velocidad previos al contacto fue la siguiente: cadera izquierda, cadera derecha, tronco, hombro izquierdo, hombro derecho, codo izquierdo, codo derecho, muñeca izquierda y muñeca derecha.

Según Lindsay y Horton ${ }^{11}$, la velocidad angular máxima del tronco en sujetos sanos es de 182 grados/s. En otro estudio realizado por Geisler ${ }^{12}$ se registraron velocidades angulares sobre el eje longitudinal de 498 grados/s en las caderas, 723 grados/s en los hombros y sobre el eje transversal de 2.090 grados/s en el palo.

Las velocidades lineales alcanzadas en la cabeza del palo de estudios previos varían en un rango que va de 40,5 a 52,6 $\mathrm{m} / \mathrm{s}^{10}$. Todos ellos se refieren a la velocidad del driver. Burden et $\mathrm{al}^{5}$ registraron con un hierro 5 velocidades máximas de 35,7 $\pm 0,4 \mathrm{~m} / \mathrm{s}$. Williams y $\mathrm{Sih}^{13}$, con el mismo palo, obtuvieron valores similares $(34,4 \pm 5,22 \mathrm{~m} / \mathrm{s})$. El criterio de rendimiento del swing de golf de la presente investigación fue la velocidad máxima alcanzada por la cara del palo.

Los objetivos de este estudio han sido: a) analizar las variables cinemáticas y la secuencia temporal durante el swing de golf, y b) hallar el patrón de movimiento en ambos grupos por sexos.

\section{MÉtodos}

La técnica de adquisición de datos es la fotogrametría 3D mediante el sistema VICON Oxford Metrics ${ }^{\circledast}$ de captura automática. Seis cámaras con focos de luz infrarroja sincronizadas registraron a 250 hercios los golpeos que se ejecutaron desde una alfombrilla de césped contra una red colocada a $5 \mathrm{~m}$ (fig. 2). Todas las sesiones de captura se desarrollaron en un control realizado por la Federación de Golf de Madrid en febrero de 2007. La muestra estaba formada por 2 jugadores y 2 jugadoras pertenecientes al Centro de Tecnificación de Golf de la Comunidad de Madrid. El hándicap del grupo era $0,3 \pm 0,6$, y la edad fue de $20,8 \pm 2,8$ años. Las chicas tenían una altura de $1,64 \pm 0,02 \mathrm{~m}$ y un peso de $53,1 \pm 1,9 \mathrm{~kg}$. Los chicos midieron $1,82 \pm 0,05 \mathrm{~m}$ y pesaron $76,8 \pm 8,8 \mathrm{~kg}$.

Cada sesión de captura fue diseñada dentro de una sesión de entrenamiento en la que también participaba su entrenador. El protocolo establecido consistió en realizar un calentamiento 
Figura I Instantes y fases del golpeo en el swing de golf.

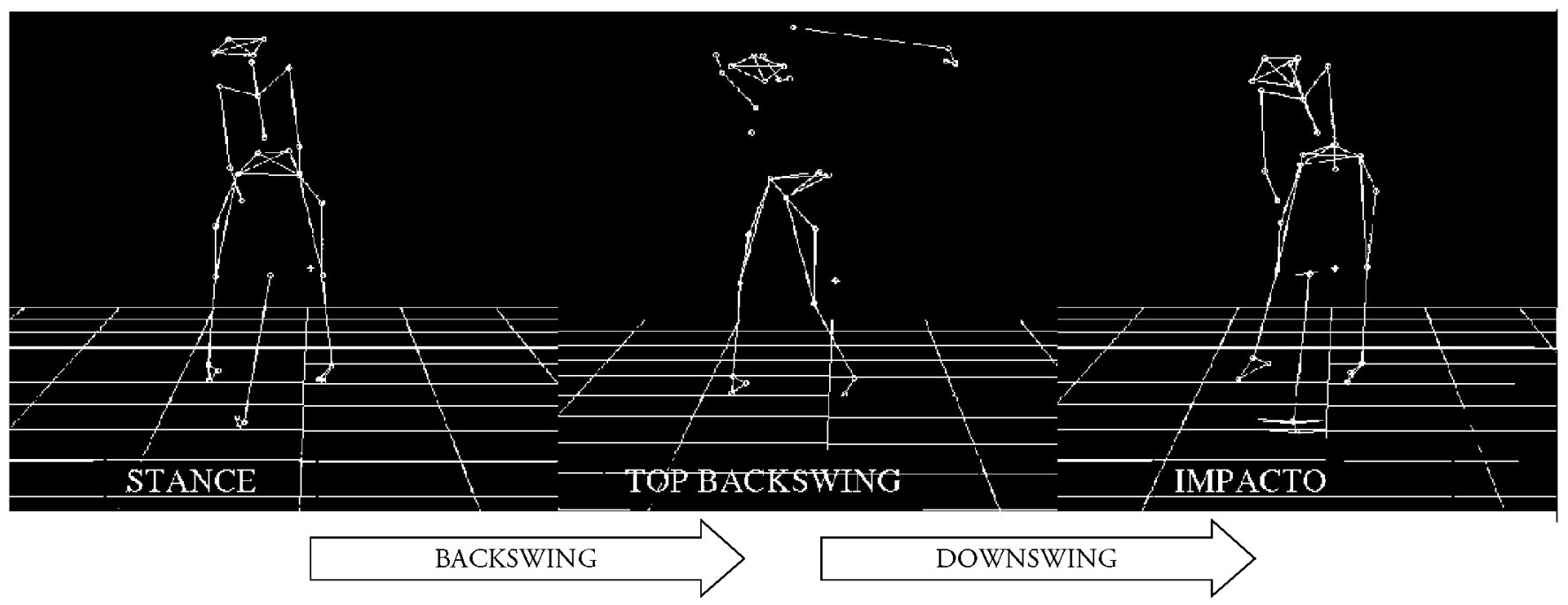

general de 15 min y luego una serie de golpeos hasta la familiarización. Se capturaron 12 golpeos consecutivos contra la red con la intención de ejecutar el mejor swing posible, es decir, dentro de su propia percepción. Las chicas eligieron un hierro 7 , mientras que los chicos eligieron un hierro 6 .

El modelo mecánico se basaba en la posición espacial de 27 marcadores reflectantes colocados en el cuerpo y 4 en el palo (fig. 3). Los marcadores fueron colocados en la punta del pie, en el maléolo externo del tobillo, en el talón, en el maléolo externo de rodilla, en el intermedio del muslo, en las crestas ilíacas anteriores y posteriores, en la apófisis subesternal, en la cavidad suprasternal, en el acromion del húmero, en el cóndilo externo del cúbito, en el intermedio del antebrazo, en la apófisis externa de la vértebra $\mathrm{C} 7,2$ en el frontal y 2 en los parietales en la zona posterior de la cabeza. En el palo se colocaron en el final del grip, en la unión de la cabeza del palo con el segmento, en el vértice superior de la cara del palo y en el punto medio de lateral de la cara del palo. Cada marcador tenía un diámetro de $14 \mathrm{~mm}$. El sistema determinaba la posición 3D de cada marcador a tiempo real a partir de las posiciones en píxeles captadas por al menos dos cámaras y a los parámetros de la transformación calculados previamente durante la calibración. La calibración efectuada mostró que el error en la posición de cada coordenada fue de menos de $2 \mathrm{~mm}$. El sistema de calibración estático determinó los ejes del sistema de referencia inercial (fig. 3).

Se registraron tanto la duración del backswing y como del downswing. Las variables cinemáticas consideradas fueron las velocidades lineales máximas de: cadera derecha, cadera izquierda, hombro izquierdo, codo izquierdo, grip y cabeza del palo, y

Figura2

Disposición de las 6 cámaras infrarrojas, plataformas y dirección de golpeo.

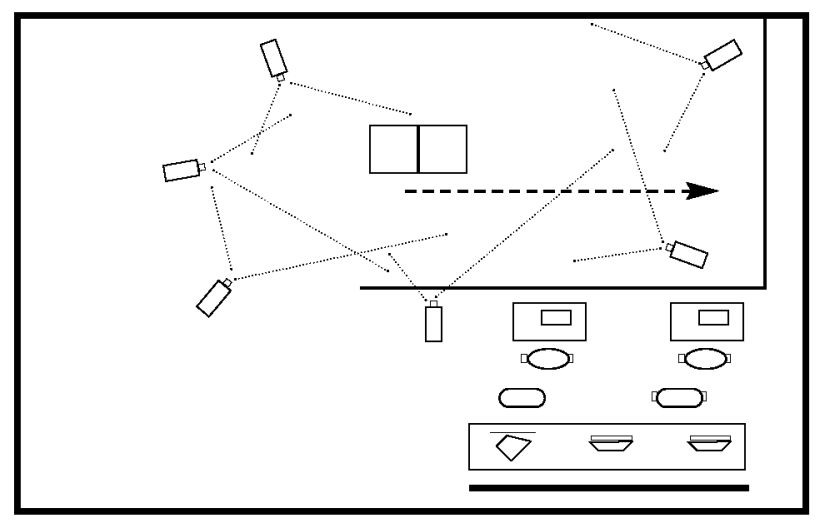

Figura 3

Sistema de referencia inercial y modelo creado.

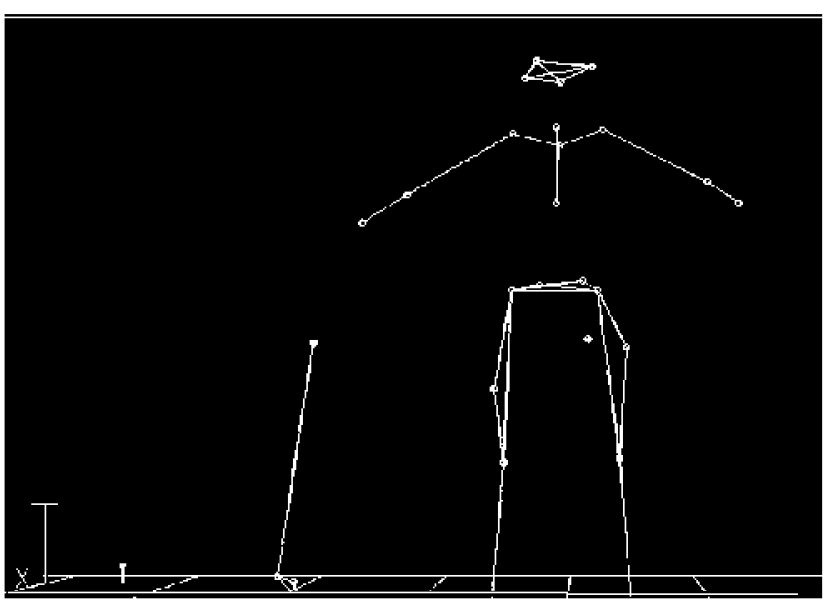


las velocidades angulares máximas de las caderas, los hombros y el palo durante la fase de aceleración downswing. Con el fin de poder realizar una comparación intersujeto e intragrupo, las variables temporales correspondientes a los tiempos máximos de las velocidades mencionadas fueron normalizados con respecto al tiempo total de la fase downswing, con el $0 \%$ en el top of backswing y el $100 \%$ en el impacto. Inicialmente se valoró considerar el mid-downswing como el $0 \%$, pero se descartó por el hecho de existir varias velocidades previas a este instante, lo que impedía normalizar en cuanto a dicho intervalo.

Para el análisis de la secuencia temporal se analizó una ANOVA de medidas repetidas de 2 factores. El factor intersujeto fue la variable sujeto, y el factor intrasujeto correspondió a las variables de los tiempos normalizados. Se aplicaron las correcciones del método Bonferroni. Se analizaron las secuencias temporales tanto de las velocidades lineales máximas como de las velocidades angulares máximas.

El criterio de rendimiento de los golpeos fue la velocidad lineal máxima alcanzada por la cara del palo. Se clasificó para cada jugador un grupo de golpeos "buenos" y otro como "malos" en función de su posición con respecto a la mediana de sus golpeos.

El análisis estadístico se realizó con el software SPSS 14.0. Con el fin de determinar si los grupos de nivel eran significativamente diferentes en cada grupo se realizó una t de Student para muestras independientes. Para analizar el rendimiento del swing en función de los parámetros biomecánicos se realizó un análisis discriminante en el que la variable dependiente era el nivel del golpeo y las variables cinemáticas y temporales fueron las variables independientes.

\section{Resultados}

Los entrenadores descartaron algunos golpeos por considerarlos de baja calidad, y la muestra final en chicas fue de $10 \mathrm{y}$ 9 golpeos para el sujeto 1 (S1) y S2, y de 7 y 11 golpeos para S3 y 54 en chicos.

Los tiempos de las fases en chicos fueron de 0,81 $\pm 0,10 \mathrm{y}$ $0,27 \pm 0,02$ s en el backswing y en el downswing, respectivamente. Las chicas golpearon en $0,99 \pm 0,08$ y $0,35 \pm 0,03 \mathrm{~s}$ en ambas fases. El tiempo total del swing fue inferior en los chicos $(1,08 \pm 0,12 \mathrm{~s})$ que en las chicas $(1,34 \pm 0,07 \mathrm{~s})$. Las desviaciones estándar demuestran que el tiempo empleado en el downswing presenta una menor variabilidad que el del backswing.

Las velocidades lineales máximas alcanzadas por las diferentes articulaciones son similares en chicas y en chicos en el codo izquierdo $(4,6$ y $5,3 \mathrm{~m} / \mathrm{s})$, en la cadera izquierda $(1,6 \mathrm{~m} / \mathrm{s}$
Tabla I Tiempos normalizados de las velocidades lineales (media $\pm \mathrm{DE}$ ) de los grupos por sexo

\begin{tabular}{|llll|}
\hline Tiempo & Chicas & \multicolumn{1}{c|}{ Tiempo } & Chicos \\
\hline Codo izquierdo & $77,3 \pm 0,6$ & Cadera izquierda & $66,2 \pm 0,8$ \\
\hline Cadera izquierda & $79,6 \pm 0,6$ & Cadera derecha* & $67,3 \pm 1,2$ \\
\hline Cadera derecha* & $81,1 \pm 1$ & Codo izquierdo* & $75,7 \pm 0,4$ \\
\hline Hombro izquierdo* & $86,2 \pm 0,7$ & Hombro izquierdo* & $81,9 \pm 0,4$ \\
\hline Palo & $95,4 \pm 0,1$ & Palo & $95,2 \pm 0,3$ \\
\hline
\end{tabular}

* Diferencias significativas $(\mathrm{p}<0,05)$, entre ese tiempo normalizado y el siguiente en la secuencia temporal.

en ambos grupos), en la cadera derecha $(1,2$ y $1,9 \mathrm{~m} / \mathrm{s})$, en el hombro izquierdo $(2,4 \mathrm{~m} / \mathrm{s}$ en ambos grupos) y en el grip $(10,6$ y $14,6 \mathrm{~m} / \mathrm{s}$ ). En cambio, la velocidad lineal máxima alcanzada en la cabeza del palo por los chicos $(38,4 \mathrm{~m} / \mathrm{s})$ es muy superior a la alcanzada por la chicas $(28,9 \mathrm{~m} / \mathrm{s})$. En cualquier caso, debe tenerse en cuenta que los chicos utilizaban un hierro 6, que, al ser de mayor longitud, imprime una mayor velocidad en la cara del palo.

El orden de las velocidades lineales varía en cuanto a la intervención del codo izquierdo, y es previo a la actuación de las caderas en el caso de las chicas y posterior a las velocidades lineales de las caderas en el grupo de los chicos. A continuación se muestran los máximos del hombro izquierdo, grip y cabeza del palo.

Las secuencias temporales de las velocidades lineales normalizadas se representan en la tabla I seleccionadas por grupos de sexo. Se observa que los tiempos de las caderas en el sexo masculino se encuentran considerablemente más distanciados del impacto que en el sexo femenino. Debido a que las velocidades máximas del grip y del palo se situaban en un intervalo muy corto de fotogramas, se decidió tomar como valor de referencia el tiempo normalizado de la velocidad máxima del palo, descartando el del grip.

A fin de comparar las muestras intersujeto e intragrupal, se realizó por separado para cada sexo una ANOVA de 2 factores (sujeto $X$ tiempos normalizados como medida repetida). En el análisis grupal de las jugadoras se observó que existían diferencias significativas $(\mathrm{p}<0,05)$ entre los tiempos del codo izquierdo $(77,3 \%)$ y el hombro izquierdo $(86,2 \%)$; entre la cadera derecha $(81,1 \%)$ y el hombro izquierdo $(86,2 \%)$, y entre la hombro izquierdo y la cabeza del palo $(95,4 \%)$. Sin embargo, no se han encontrado diferencias significativas entre los tiempos del codo izquierdo $(77,3 \%)$ y la cadera izquierda 
Tabla II

Velocidades angulares máximas registradas en grados/segundo

\begin{tabular}{|lcr|}
\hline $\begin{array}{l}\text { Velocidad angular } \\
\text { máxima }\end{array}$ & $\begin{array}{c}\text { Chicas } \\
\text { (media } \pm \text { DE) }\end{array}$ & $\begin{array}{c}\text { Chicos } \\
\text { (media } \pm \text { DE) }\end{array}$ \\
\hline Cadera & $452,0 \pm 60,7$ & $540,6 \pm 53,5$ \\
\hline Hombro & $717,6 \pm 98,1$ & $758,9 \pm 42,2$ \\
\hline Palo & $1.737,3 \pm 35,9$ & $2.233,7 \pm 61,1$ \\
\hline
\end{tabular}

$(79,6 \%)$, ni entre la cadera izquierda y la cadera derecha $(81,1 \%)$ (tabla I). En el análisis en conjunto del grupo masculino se hallaron diferencias significativas $(\mathrm{p}<0,05)$ entre la cadera derecha $(67,3 \%)$ y el codo izquierdo $(75,7 \%)$; entre el codo izquierdo y el hombro izquierdo $(81,9 \%)$, y entre el hombro izquierdo y la cabeza del palo $(95,2 \%$ ) (tabla I).

Las velocidades angulares máximas alcanzadas se muestran en la tabla II. Al igual que en las velocidades lineales, la velocidad angular de la cabeza del palo es considerablemente superior en los chicos respecto a las chicas.

Las secuencias temporales de los instantes en que ocurrían dichos picos máximos de velocidades angulares normalizados dentro del downswing se muestran en la tabla III, con el $0 \%$ en el instante del top of backswing y el 100\% en el impacto. Los tiempos normalizados muestran que las tres acciones valoradas se encuentran más diferenciadas en el tiempo en el caso de los chicos. En el análisis grupal de estos tiempos en ambos grupos, masculino y femenino, existían diferencias significativas $(p<0,05)$ entre el instante normalizado y el siguiente en la secuencia temporal.

En la comparación de cada secuencia temporal de cada jugador, entre S1 y S2, y entre S3 y S4, en ambos casos cada jugador mantuvo una secuencia significativamente diferente respecto al otro sujeto comparado (figs. 4 y 5).

Para establecer los niveles de los golpeos se tomó como criterio de referencia la velocidad máxima de la cabeza del palo. La mediana de cada grupo fue tomada como límite entre los niveles. El nivel 1 fueron los golpeos considerados como "buenos", mientras que el nivel 2 fueron los golpeos "malos". Se realizó una t de Student para muestras independientes, que estableció que existían diferencias significativas entre ambos grupos del nivel de $\mathrm{p}<0,01$.

A fin de determinar en qué medida las variables analizadas influían en el rendimiento del golpeo se realizó un análisis estadístico desde 2 puntos de vista: uno basado en las variables cinemáticas que tenían una secuencia temporal más estable, es decir, en las velocidades angulares máximas como variables in-
Tabla III Tiempos normalizados de las velocidades máximas angulares (media $\pm \mathrm{DE}$ ) de los grupos por sexo

\begin{tabular}{|lcc|}
\hline Tiempo & Chicas & Chicos \\
\hline \% T velocidad angular cadera* & $73,1 \pm 0,7$ & $62,6 \pm 1,1$ \\
\hline \% T velocidad angular hombro* & $82,8 \pm 0,6$ & $77,4 \pm 0,4$ \\
\hline \% T velocidad angular palo* & $94,8 \pm 0,2$ & $94,3 \pm 0,2$ \\
\hline
\end{tabular}

* Diferencias significativas $(P<0,05)$ entre ese tiempo normalizado y el siguiente en la secuencia temporal, en las velocidades angulares máximas alcanzadas.

\% T: porcentaje de tiempo normalizado en el downswing.

dependientes, y el segundo basado en "cuándo" ocurrían dichas velocidades angulares máximas dentro del dowswing, es decir, los tiempos normalizados como variables independientes, y en ambos grupos la variable dependiente fue el nivel del golpeo.

En el análisis discriminante de las velocidades angulares máximas y su relación con el rendimiento se hallaron fórmulas significativas en ambos grupos de sexo. En el grupo de los chicos el nivel de significación fue de $\mathrm{p}<0,001$. Esta fórmula discriminante $o F(d)$ indica que el nivel 1 ("golpeos buenos") se produce cuando el resultado de la misma es positivo, y es del nivel 2 ("golpeos malos") cuando es negativa. Puede predecir un $94,4 \%$ de los casos.

$F(d)=0,037 \times$ velocidad angular máxima caderas -

$0,011 \times$ velocidad angular máxima hombros $-11,685$

Coeficiente de correlación canónica: 0,813

$\mathrm{p}<0,001$

Acierto de casos: $94,4 \%$

Según esta fórmula, los swings "buenos" se producirán cuando la fórmula discriminante sea positiva, y para ello las caderas han de obtener una gran velocidad angular, y por lo tanto sería el elemento que habría que potenciar.

$F(d)=0,007 \times$ velocidad angular máxima caderas +

$0,009 \times$ velocidad angular máxima hombros $-9,254$

Coeficiente de correlación canónica: 0,617

$\mathrm{p}<0,05$

Acierto de casos: $78,9 \%$

En el caso de las chicas, la fórmula discriminante obtuvo un nivel de significación de $\mathrm{p}<0,05$, con un acierto del 78,9\% de los casos. Los swings "buenos" resultaban positivos al aplicar la fórmula, mientras que los swing "malos" eran negativos. 
Figura 4 Secuencia de los tiempos normalizados de las velocidades lineales en los sujetos I, 2, 3 y 4.
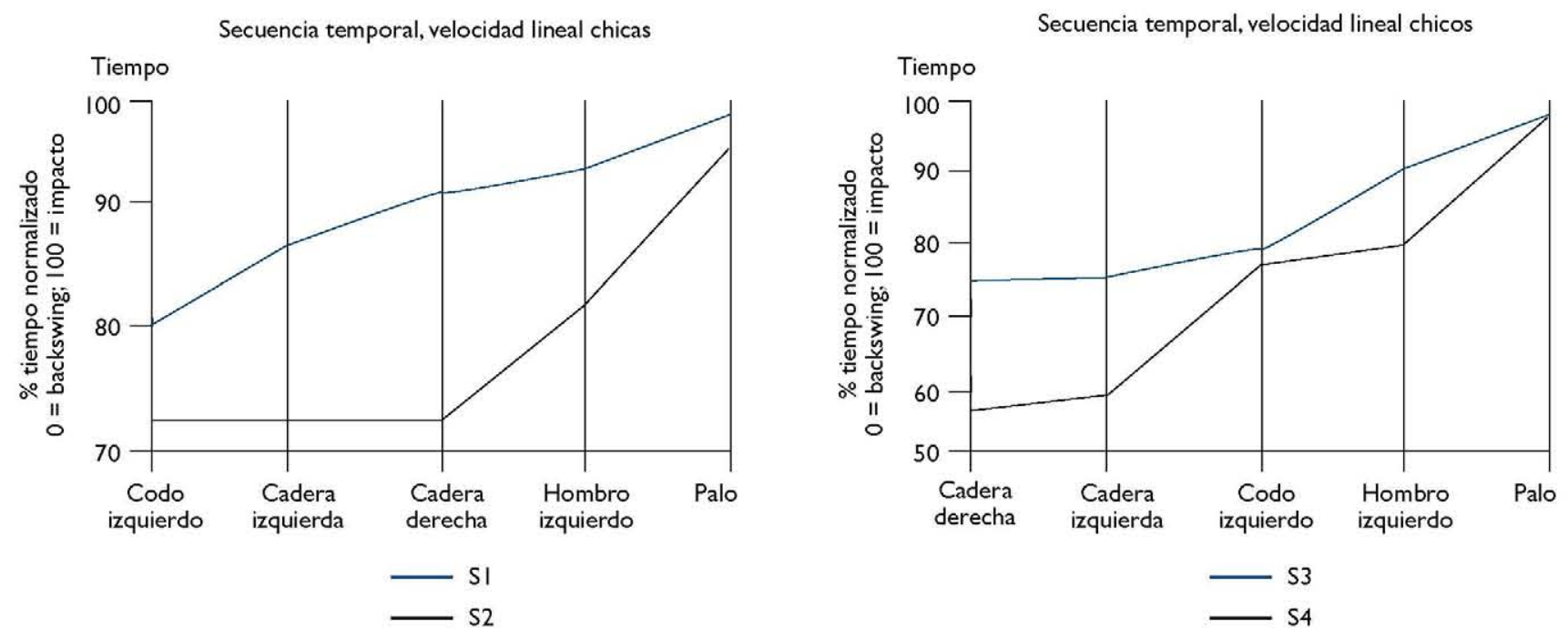

Figura 5 Secuencias temporales de las velocidades angulares de los sujetos I, 2, 3 y 4.

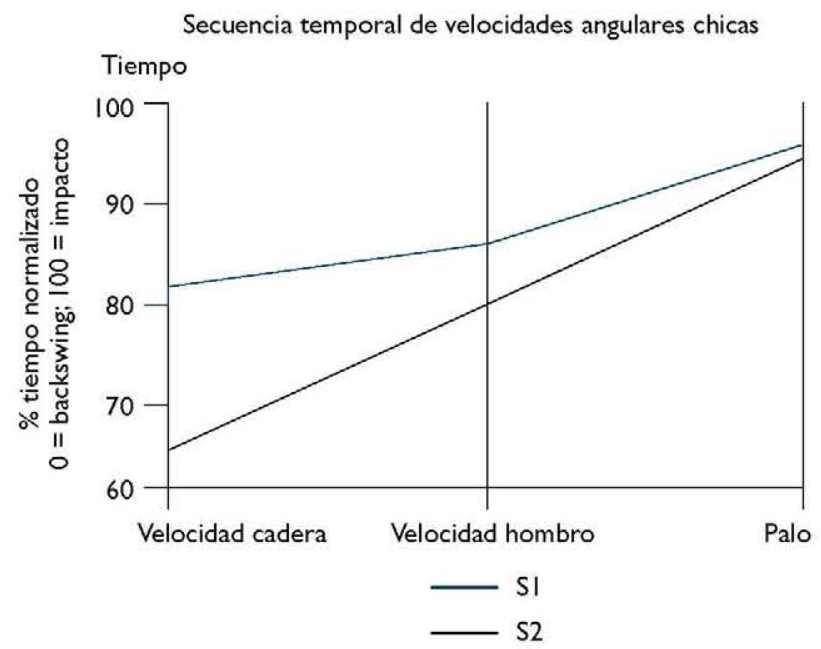

La interpretación de esta fórmula discriminante indica que habría que potenciar ambas velocidades angulares para obtener un "buen" swing, y que la velocidad angular lograda por las caderas tenía ligeramente más peso en dicho resultado final.

La segunda línea del análisis discriminante se basó en analizar "cuándo" se producían las velocidades angulares máximas, es decir, en discriminar si los swing "buenos" dependen de una buena "coordinación" de dichas acciones segmentarias. Se halló una fórmula discriminante en el caso de los chicos, pero no en el grupo de las chicas.

La fórmula discriminante de los tiempos normalizados de

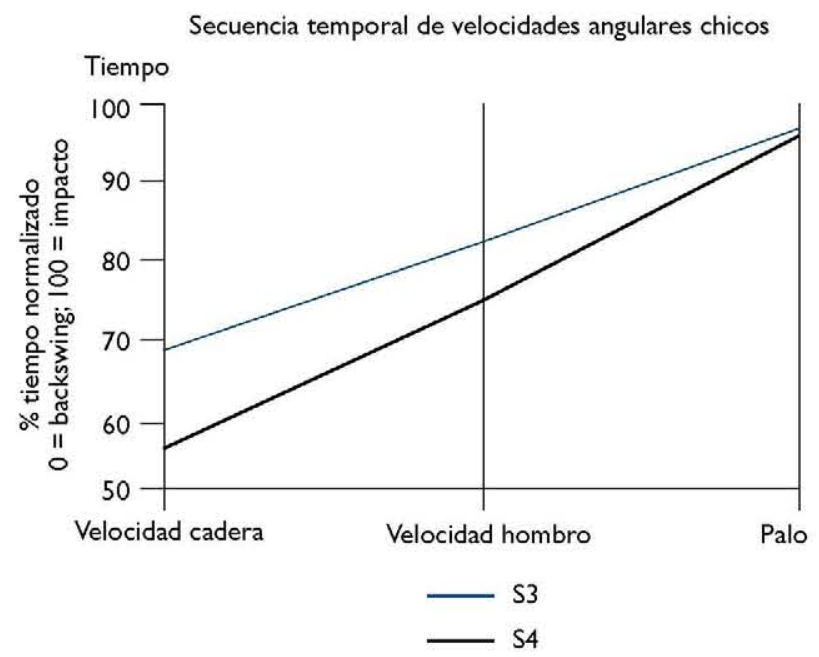

las velocidades angulares máximas tenía una significación de $\mathrm{p}<0,005$.

$F(d)=0,034 \times \%$ tiempo normalizado en el downswing caderas - $0,516 \times \%$ tiempo normalizado en el downswing hombros $+38,104$

Correlación canónica: 0,754

$\mathrm{p}<0,005$

Acierto de casos: $88,9 \%$

En esta fórmula los resultados positivos son considerados del grupo 1 ("buenos"), mientras que los negativos se conside- 
ran del grupo 2 ("malos"). Dada la media de ambos tiempos normalizados, que es de $62,6 \%$ en el tiempo de las caderas y de $77,4 \%$ en el de los hombros, y el valor de la constante de la fórmula $(38,1)$, ambos datos indican que existe un rango óptimo de coordinación en el que, cuando las caderas actúan de forma más temprana, el swing tendrá un mejor rendimiento.

\section{Discusión}

La duración de las fases del golpeo backswing en el grupo masculino $(0,81 \pm 0,11$ s) y downswing $(0,27 \pm 0,02$ s) encontrados son similares a los de otros estudios previos ${ }^{4,7,14-15}$, en el caso del sexo masculino. En las chicas, el backswing $(0,99 \pm$ $0,08 \mathrm{~s}$ ) presenta un valor más alto que en otros estudios, aunque creemos que esta diferencia no se debe a razones antropométricas sino posiblemente al mejor nivel técnico de la muestra empleada en el presente estudio.

Las velocidades lineales de los estudios fundamentados en el hierro 5 (el palo más similar a hierro 6 y hierro 7 ) son inferiores a los valores alcanzados por los chicos ${ }^{6,10}$. De nuevo nos inclinamos a pensar que esta discrepancia se debe a que el nivel de rendimiento de nuestra muestra es considerablemente superior que el de los estudios consultados.

Nesbit y Serrano ${ }^{10}$ establecieron una primera secuencia temporal basada en el trabajo de los diferentes segmentos. Sólo en el caso del jugador con mejor hándicap (0-scratch) el impacto era posterior a dichos máximos, lo que coincide con lo encontrado en nuestro estudio, donde el máximo de velocidad del palo se produce un 5\% antes del impacto con relación con el tiempo total del downswing. La secuencia encontrada para los chicos demuestra que el movimiento empieza por las caderas, sigue por el codo y el hombro izquierdo, y es la cabeza del palo la que llega a su máximo en último lugar y ligeramente antes del impacto. Esta secuencia coincide con la descrita por Nesbit y Serrano ${ }^{10}$, salvo que estos autores han encontrado que los hombros llegan al máximo antes que los codos. Esta diferencia de resultados puede deberse al tipo de variable utilizada, dado que estos autores tomaron el trabajo mecánico realizado como parámetro fundamental. En cualquier caso, es importante remarcar que en nuestro estudio se han utilizado 2 muestras (chicos y chicas), y que en ambos casos el máximo del codo izquierdo se produce antes que el máximo del hombro izquierdo, diferencia que es de $6,54 \%$ (de la fase de aceleración) en los chicos y del $5,11 \%$ en las chicas. Es relevante precisar que en el grupo de chicas no se ha podido confirmar que las caderas actúan antes que el codo; en concreto, en las dos jugadoras analizadas el máximo de velocidad de la cadera derecha fue poste- rior al del codo izquierdo. Podríamos, en principio, pensar que ello se debe a la falta de fuerza en el miembro inferior que impide mantener el stance, aunque consideramos que este dato debe confirmarse con otros estudios. En cuanto a la actuación de las caderas, ni en los chicos ni en las chicas se han encontrado diferencias significativas entre la actuación de la cadera derecha y la izquierda.

Las velocidades angulares registradas (tabla III) son similares al estudio de Geisler ${ }^{12}$. Resulta llamativo que la velocidad angular alcanzada en la cabeza del palo en los chicos (2.233,7 grados/s) sea considerablemente superior al dato de dicho estudio (2.090 grados/s). Este hecho lo atribuimos al excelente nivel de la muestra considerada. En el caso de la chicas, el registro de 1.737 grados/s no debe considerarse de baja calidad, ya que aunque se desconoce la muestra del estudio de Geisler ${ }^{12}$, los sujetos de estudio suelen ser masculinos, con lo que este dato inferior podría deberse a una cuestión intrínseca de las capacidades de cada sexo.

La secuencia temporal de velocidades angulares no ha sido considerada en estudios previos, por lo que las diferencias significativas en ambos grupos muestran la estabilidad de la secuencia temporal de cada sujeto en la que actúa primero el giro de caderas, posteriormente los hombros y por último el palo.

Es importante remarcar la no existencia de estudios que investiguen la relación entre la secuencia de movimientos y el rendimiento final. Los resultados encontrados en este estudio permiten asegurar que el empleo de técnicas estadísticas de clasificación de patrones de movimiento tales como el análisis discriminante pueden utilizarse a la hora de abordar el rendimiento técnico individual.

El análisis discriminante efectuado en función del sexo ha permitido establecer las claves en cuanto a qué acción segmentaria es más importante en el rendimiento del swing. En el caso de los chicos se ha podido demostrar que el rendimiento mejora cuando aumenta la velocidad angular máxima de las caderas y cuando este máximo se produce distanciado en el tiempo respecto a la máxima velocidad angular de los hombros.

En las chicas, las acciones del giro de caderas, del giro de hombros y la bajada del palo aparecen más agrupadas en el tiempo, por lo que se puede indicar que golpean más "todo en uno". Este hecho que indica que las acciones ocurren de forma más agrupada en el tiempo puede ser la razón por la que no existe una fórmula discriminante en relación al tiempo normalizado.

Las diferencias relativas a "cuándo" se producen estos giros podrían ser la clave para alcanzar una mayor velocidad en la cabeza del palo, por lo que sería recomendable, en el caso de las 
chicas, disociar en el tiempo ambos giros con el fin de producir un swing más potente.

\section{ConClusiones}

Se ha puesto a punto un sistema para análisis del swing de golf que permite dar información rápida al entrenador y deportista. Se ha encontrado que la secuencia de movimientos lineales empieza por las caderas y sigue por el codo izquierdo, el hombro izquierdo y los extremos del palo en los chicos. Las chicas actúan primero con el codo izquierdo y simultáneamente con las caderas, para finalmente actuar con el hombro izquierdo y el palo. Las secuencias de las velocidades angulares de todos los sujetos seguían el mismo patrón: giro de caderas, giro de hombros y aceleración del palo.

\section{Bibliografía}

1. Kreighbaum E, Barthels KM. Biomechanics. A qualitative approach for studying human movement. Minneapolis, Minnesota: Burgess Publishing Co; 1981.

2. Hume PA, Keogh J, Reid D. The role of biomechanics in maximising distance and accuracy of golf shots. Sports Med. 2005; 35:429-49.

3. Ball KA, Best RJ. Different centre of pressure patterns within the golf stroke I: cluster analysis. J Sports Sci. 2007;25:757-70.

4. Cochran A, Stobbs J. The search for the perfect golf swing. 1st ed. Philadelphia: J.B. Lippincott; 1968.

5. Burden AM, Grimshaw PN, Wallace ES. Hip and shoulder rotations during the golf swing of sub-10 handicap players. J Sports Sci. 1998;16:165-76.

6. Milburn PD. Summation of segmental velocities in the golf swing. Med Sci Sports Exerc. 1982;14:60-4.

7. Neal RJ, Wilson BD. 3D kinematics and kinetics of the golf swing. International Journal of Sports Biomechanics. 1985; $1: 221-32$.
Dentro de estos patrones generales para cada sexo, se han encontrado diferencias entre los jugadores, lo que demuestra la existencia de una técnica individual que debe tenerse en cuenta a la hora del valorar el rendimiento técnico. Las velocidades angulares serían más adecuadas para realizar un análisis biomecánico, ya que se trata de un golpeo basado más en giros que en desplazamientos.

La coordinación de las acciones es la clave determinante en el rendimiento de este tipo de golpeos. Mediante el análisis discriminante se ha puesto de manifiesto que el rendimiento de los golpeos está relacionado directamente -además de con la velocidad de giro- con el tiempo en el que ocurre dicha acción.

Dado que la muestra es de gran nivel y de jugadores promesas, se propone este tipo de estudios como controles periódicos dentro de la planificación del entrenamiento.

8. Coleman S, Anderson D. An examination of the planar nature of the golf club motion in the swings of experience players. J Sports Sci. 2007;25:739-48.

9. Egret C, Leroy D, Chollet D, Loret A, Weber J. Approche scientifique du swing. Education Physique et Sportive. 2000; 286:61-5.

10. Nesbit SM, Serrano M. Work and power analysis of the golf swing. Journal of Sports Science and Medicine. 2005;4:520-33.

11. Lindsay D, Horton J. Comparison of spine motion in elite golfers with and without low back pain. J Sports Sci. 2002;20:599-605.

12. Geisler PR. Golf. En: Shamus E, Shamus J, editors. Sports injury prevention and rehabilitation. New York: McGraw-Hill; 2001.

13. Williams KR, Sih BL. Changes in golf clubface orientation following impact with the ball. Sports Engineering. 2002;5:65-80.

14. Egret CI, Nicolle B, Dujardin FH, Weber J, Chollet D. Kinematic analysis of the golf swing in men and women experienced golfers. Int J Sports Med. 2006;27:463-7.

15. Coleman SG, Rankin AJ. A three-dimensional examination of the planar nature of the golf swing. J Sports Sci. 2005;23:227-34. 